\title{
Comparison of Feasibility and Safety of Epidural Catheterization between Cranial and Caudal Lumbar Vertebral Segments in Dogs
}

\author{
Di ZHANG ${ }^{1 *}$, Ryohei NISHIMURA ${ }^{1)}$, Shotaro NAGAHAMA ${ }^{2)}$, Toshie ISERI ${ }^{4)}$, Manabu MOCHIZUKI ${ }^{3)}$, \\ Takayuki NAKAGAWA ${ }^{3)}$ and Nobuo SASAKI ${ }^{3)}$ \\ ${ }^{1)}$ Laboratory of Veterinary Emergency Medicine, ${ }^{2)}$ Veterinary Medical Center and ${ }^{3)}$ Laboratory of Veterinary Surgery, Graduate School \\ of Agricultural and Life Sciences, The University of Tokyo 1-1-1 Yayoi, Bunkyo-ku, Tokyo 113-8657 and ${ }^{4)}$ Department of Advanced \\ Clinical Medicine, Graduate School of Life and Environmental Sciences, Osaka Prefecture University, Osaka 598-8531, Japan
}

(Received 7 April 2011/Accepted 15 July 2011/Published online in J-STAGE 29 July 2011)

\begin{abstract}
To compare the technical difficulty and safety of epidural catheterization between cranial and caudal lumbar region, thirteen dogs were randomly assigned to a cranial lumbar group (group CraL, $n=6$ ) or a caudal lumbar group (group CauL, $n=6$ ) depending on different epidural sites, and one dog was used as a negative control without catheterization. After general anesthesia, an epidural catheter was advanced $10 \mathrm{~cm}$ cranially from the interspace of L1-L2 in group CraL or from lumbosacral space in group CauL. Dogs were euthanized and catheter position and tip location were confirmed by laminectomy. Spinal cord samples were examined by macro- and microscopic observations. Success rate, time taken for epidural space confirmation and catheter insertion were compared, and overall technical difficulty was evaluated subjectively. Epidural catheter was inserted successfully in all dogs. Time needed from needle skin puncture to catheter placement and saline injection was $226 \pm 63$ and $229 \pm 26 \mathrm{sec}$ in groups CraL and CauL without significant differences. Three dogs in group CraL suffered subcutaneous blood, but no spinal cord injuries were found. Subjective evaluation score of the overall technical difficulty was slightly but significantly higher in group CraL than in group CauL $(P=0.009)$. Epidural catheterization in cranial lumbar region could be performed as feasible and safe as that at the caudal lumbar vertebral region in medium or large dogs. KEY WORDS: canine, catheterization, epidural anesthesia, lumbar vertebrae, safety.
\end{abstract}

In human medicine, neuroaxial blockades are commonly used intra- and postoperatively for providing potent segmental anesthesia and analgesia. Epidural needles and catheters can be inserted into various intervertebral levels depending on the intended surgical procedure. Thoracic epidural administration, especially by constant infusion of drugs, has been reported to provide superior anesthesia and analgesia for thoracic, cardiac, and upper abdominal surgeries and to improve the post-operative outcome [3].

In dogs, lumbarsacral epidural anesthesia and analgesia is usually provided at caudal lumbar vertebral segments, which is the most frequently used neuroaxial block for surgical procedures caudal to the umbilicus [16]. Although experimental studies on thoracic epidural analgesia have been reported $[6-8,13]$, epidural neuroaxial blocks in the thoracic or cranial lumbar area have rarely been used in the clinical setting. However, a paper documented an easy access at T13-L1 and L1-L2 levels for the injection of a contrast medium for myelography [15]; therefore, we believe that needle insertion and epidural catheterization should be feasible through the cranial lumbar intervertebral spaces.

The aim of the present study was to compare the technical difficulties and technique-related complications of epidural catheterization in cranial lumbar vertebral segments with

\footnotetext{
* Correspondence to: Zhang, D., Laboratory of Veterinary Emergency Medicine, Graduate School of Agricultural and Life Sciences, The University of Tokyo, 1-1-1 Yayoi, Bunkyo-ku, Tokyo 113-8657, Japan.

e-mail: vetzhang101@hotmail.com
}

those in the caudal lumbar vertebral (lumbosacral) segments.

\section{MATERIALS AND METHODS}

This study was approved by the Ethics Committee of Animal Use and Care Committee of the Graduate School of Agricultural and Life Sciences, The University of Tokyo. Thirteen healthy male beagles with a mean age of 48.3 months (range from 37 to 82 months) and a mean body weight of $13.0 \mathrm{~kg}$ (range from 11.2 to $15.5 \mathrm{~kg}$ ) were used in this study. Each dog was used for one treatment. All dogs were housed in individual cages maintained at constant temperature and humidity. Food was withheld for at least $12 \mathrm{hr}$ before the experiment.

Dogs were randomly assigned to 2 groups depending on the epidural catheter insertion site: cranial lumbar vertebra group (group CraL) and caudal lumbar vertebra group (group CauL). Each group consisted of 6 dogs; one dog was not catheterized and was used as a general anesthesia and anatomical control. Mask induction with isoflurane (Escain; Mylan Inc., Osaka, Japan) followed by endotracheal intubation, and anesthetic maintenance with isoflurane diluted in $100 \% \mathrm{O}_{2}$ were performed in all dogs. After endotracheal intubation, dogs were positioned in sternal recumbency with their limbs pulled forward. Hair was clipped, and the skin surface surrounding the intended needle puncture site was prepared using the standard surgical preparation procedure. An 18-G Tuohy needle and a 20-G radiotransparent flexible catheter (Hakko Co. Ltd., Nagano, 
Japan) were used for epidural catheterization. All procedures of epidural needle and indwelling catheter insertion were performed by one investigator (SN) who had gained experience by practicing on cadavers before this experiment and could perform the procedure without any auxiliary equipment. The needle puncture site on the skin (the intervertebral space between $\mathrm{T} 1$ and $\mathrm{T} 2$ in group $\mathrm{CraL}$ and between $\mathrm{L} 7$ and $\mathrm{S} 1$ in group $\mathrm{CauL}$ ) was determined by palpating the iliac wings and the dorsal spinous process of L7, and by counting the dorsal spinous processes of lumbar segments cranially up to the required site.

In group CraL, the Tuohy needle was inserted into the epidural space via a paramedian approach. The skin puncture site was on the right or left side with respect to the spinous process and slightly posterior to the predetermined intervertebral space. After penetrating the subcutaneous tissue, the Tuohy needle was advanced anteroventrally with its bevel facing cephalad. Correct placement of the needle in the epidural space was confirmed by "loss of resistance (LOR)" technique with saline. If there was no LOR, needle was redirected or a second puncture was performed. Each new skin puncture was recorded as another attempt, regardless of whether it was at the predetermined vertebral level or at an adjacent vertebral level (anterior or posterior to the predetermined one). Simply redirecting the needle without a new skin puncture was not counted as an additional attempt. A successful placement of the epidural needle was identified by a positive LOR. After confirmation of correct needle placement, an epidural catheter was introduced through the needle and advanced $10 \mathrm{~cm}$ into the epidural space. After needle withdrawal, the catheter was aspirated using a syringe to confirm no blood or cerebrospinal fluid. The catheter was then secured to the skin with a butterfly tape and suture. In group CauL, epidural catheterization was performed via the midline approach. The Tuohy needle was placed perpendicular to the skin surface at the centre of the intervertebral space. Needle puncture, epidural space confirmation, catheter insertion, and other procedures were identical to those in group CraL. A volume of saline $(0.2$ $\mathrm{ml} / \mathrm{kg}$ ) was injected through the catheter within $2 \mathrm{~min}$ in both groups. The time taken from needle skin puncture to epidural space confirmation and the time from needle skin puncture to epidural catheter placement and saline injection were recorded. The depth of needle inserted (from skin puncture site to the needle tip) was also recorded by checking the mark on the needle. The overall procedure was evaluated subjectively by one investigator ( $\mathrm{SN}$ ) according to a composite subjective evaluation scale (Table 1).

After epidural injection of saline, all dogs were euthanized with an intravenous injection of thiopental sodium (70 $\mathrm{mg} / \mathrm{kg}$, Ravonal ${ }^{\circledR}$; Mitsubishi Tanabe Pharma Corporation, Osaka, Japan) along with isoflurane anesthesia to ensure deep anesthesia, which was confirmed by the absence of corneal reflexes and muscle tone; this was followed by intravenous injection of potassium chloride $(300 \mathrm{mg} / \mathrm{kg}$, $\mathrm{KCl}$ drip injection 15\%; Maruishi Pharmaceutical Co. Ltd., Osaka, Japan). Catheter position and tip location were confirmed by an epidurography using iohexol (Omnipaque 300; Daiichi Seiyaku Co. Ltd., Tokyo, Japan). The number of spinal bodies contacted by the catheter was recorded by using a modified method based on a previous report [10]: a complete segment which was contacted by the catheter was counted as 1 vertebral body unit (VBU), and part of a segment was counted as $0.5 \mathrm{VBU}$. The control dog was also euthanized and subjected to radiography.

After radiography, the catheter within the epidural space and the spinal cord were exposed and inspected macroscopically by excising vertebral arches. Data were recorded for catheter tip side with respect to the spinal cord, techniquerelated tissue injuries, including tissue bleeding, dural puncture, and canalization. In addition, spinal cord segment samples: (1) around the tip of the catheter, (2) around the needle puncture site, and (3) from the middle of these 2 sites were collected. Tissue samples were fixed in $10 \%$ neutral formalin solution, mounted into paraffin sections, and stained with hematoxylin and eosin (HE) for microscopic evaluation.

Table 1. A composite subjective evaluation scale

\begin{tabular}{|c|c|c|}
\hline Item & Criteria & Score \\
\hline \multirow{3}{*}{$\begin{array}{l}\text { Determination of } \\
\text { skin puncture site }\end{array}$} & $\begin{array}{l}\text { The landmark can be palpated obviously and } \\
\text { the puncture site can be determined easily }\end{array}$ & 1 \\
\hline & $\begin{array}{l}\text { The landmark can be palpated and the } \\
\text { puncture site can be determined }\end{array}$ & 2 \\
\hline & $\begin{array}{l}\text { The landmark is unclear, and the puncture site } \\
\text { can not be determined by palpating landmarks }\end{array}$ & 3 \\
\hline \multirow{3}{*}{$\begin{array}{l}\text { Needle puncture } \\
\text { attempt }\end{array}$} & $\begin{array}{l}\text { Needle can be inserted into the epidural space } \\
\text { at the first attempt without needle redirection }\end{array}$ & 1 \\
\hline & $\begin{array}{l}\text { Needle can be inserted into the epidural space } \\
\text { within two attempts after needle redirection }\end{array}$ & 2 \\
\hline & $\begin{array}{l}\text { Needle cannot be inserted into the epidural } \\
\text { space after three attempts }\end{array}$ & 3 \\
\hline \multirow{3}{*}{$\begin{array}{l}\text { Resistance of } \\
\text { catheterization }\end{array}$} & Mild, catheter can be inserted evenly & 1 \\
\hline & Moderate, catheter can be inserted & 2 \\
\hline & Catheter cannot be inserted & 3 \\
\hline
\end{tabular}


Normality of the data distribution was assessed initially using the $\chi^{2}$ test for goodness of fit. Time-related variables and needle insertion depth were compared between the 2 groups by unpaired $t$-test. The subjective evaluation and epidural catheter position were analyzed by Mann-Whitney $U$ test. A significant level of $P<0.05$ was used for all statistical tests.

\section{RESULTS}

Epidural catheterization was successfully at the first attempt in 3 of the 6 dogs in group CraL and 5 of the 6 dogs in group CauL. In the remaining dogs ( 3 dogs in group CraL and 1 dog in group CauL), successful puncture and catheterization were performed at the second attempt. There was no significant difference either in time from needle skin puncture to epidural space confirmation $(P=0.445)$ or time from needle skin puncture to epidural catheter insertion and saline injection $(P=0.907)$ between group $\mathrm{CraL}$ and group $\mathrm{CauL}$ (Table 2).

The results of epidural catheter position and tip location are shown in Table 3. The needle length from the skin surface to the epidural space was longer in group $\mathrm{CraL}$ than in group CauL. Actual needle puncture point on the skin surface in group CraL was L1-L2 in 5 dogs and L2-L3 in 1 dog (Fig. 1); in group CauL, it was L6-L7 in 3 dogs and L6-S1 in the other 3 dogs. The median number of vertebral segments contacted by the epidural catheter was 3 in each group. There was no difference of catheter tip location with respective to the spinal cord between the 2 groups $(P=0.240)$.
Subcutaneous bleeding around the needle puncture site was detected in 3 dogs in group CraL but in no dog in group CauL. There were no macroscopic injuries to the spinal cord tissue in the control dog or in any of the cathetertreated dogs. No significant histopathological changes were observed in the control dog or in the catheter-treated dogs.

Results of subjective evaluation are shown in Table 4 . The score of the overall technical difficulty was slightly but significantly higher in group $\mathrm{CraL}$ than group $\mathrm{CauL}$ $(P=0.009)$.

\section{DISCUSSION}

In dogs, epidural anesthesia is commonly provided in lumbosacral space for surgical procedures involving regions caudal to the umbilicus [16]. Epidural injection of local anesthetics in more cranial area is expected to provide preferred analgesia for surgical procedures in more cranial regions, such as thoracotomy or upper abdominal surgery. However, needle puncture through the intervertebral spaces of thoracic vertebrae is believed to be technically difficult or clinically risky. Therefore, we proposed the placement of the epidural needle through the intervertebral space between L1 and L2, as in group CraL in this study.

We identified the puncture site by means of palpating the iliac crest and spinous processes, however, the actual epidural puncture site in either group $\mathrm{CraL}$ or group $\mathrm{CauL}$ was not always in concordance with our prediction. In clinical studies, body weight or body habitus is of a high variability among individuals, which may possibly affect the quality of landmarks, consequently affect the result of the correct

Table 2. Time (sec) consumed from needle skin puncture until epidural space confirmation, and until epidural catheter insertion and saline injection

\begin{tabular}{lcc}
\hline Time consumed from needle skin puncture & Group CraL $(\mathrm{n}=6)$ & Group CauL $(\mathrm{n}=6)$ \\
\hline Until epidural space confirmation & $90 \pm 44$ & $74 \pm 19$ \\
Until epidural catheter insertion and saline injection & $226 \pm 63$ & $229 \pm 26$ \\
\hline
\end{tabular}

There were no significant differences between two groups. Values shown as mean \pm SD.

Table 3. Epidural catheter position and tip location in the epidural space in CraL and CauL groups

\begin{tabular}{lcc}
\hline & Group CraL (n=6) & Group CauL (n=6) \\
\hline Needle insertion depth (cm; median, min-max) & $4,3-5^{*}$ & $3,2-4$ \\
Actual puncture site (number of dogs) & L1/L2 (5) & L6/L7 (3) \\
& L2/L3 (1) & L7/S1(3) \\
& T11 (2) & L3/L4(2) \\
Catheter tip location (number of dogs) & T11/T12(1) & L4 (2) \\
& T12 (1) & L5 (2) \\
Number of vertebral column segments contacted & T12/T13 (2) & \\
$\quad$ by epidural catheter (median, min-max) & $3,2-3.5$ & $3,1.5-3.5$ \\
Catheter tip side (number of dogs) & R:M:L=5:1:0 & R:M:L=4:0:2 \\
\hline
\end{tabular}

R: right side to the spinal cord; M: dorsal lateral to the spinal cord; L: left side to the spinal cord.

* Statistically different between two groups $(P=0.039)$. 


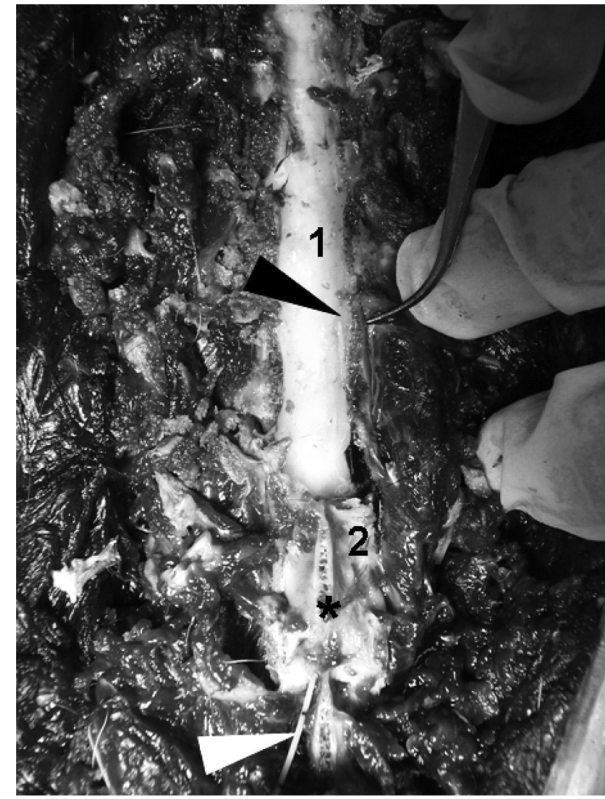

Fig. 1. Photograph of cranial lumbar spinal cord (vertebral arch was partly removed). The epidural catheter was inserted into the epidural space from L1 and L2 interspace (black arrow: the tip of the catheter; white arrow: catheter located in the subcutaneous tissues; asterisk: stubbed spinous process of L1; 1: spinal cord; 2: the vertebral body of L1).

Table 4. Subjective evaluation of the overall technical difficulty in group $\mathrm{CraL}$ and group CauL

\begin{tabular}{|c|c|c|}
\hline & Group CraL & Group CauL \\
\hline Subjective evaluation & $5(4,6)^{*}$ & $3(3,5)$ \\
\hline
\end{tabular}

placement of the needle tip in the epidural space at the first chosen site $[4,17]$. However, in our study, we used standard experimental dogs, in which body weight changed within a narrow range. Hence the present unintended epidural site was unlikely related to the body weight. We consider that the accuracy of epidural puncture at the predicted site could be enhanced after more practice.

All epidural puncture and catheterization was performed by the only one investigator. The selection of epidural approach was determined by his own experience and interest, whereas, we thought it was reasonable to use the paramedian approach for catheter insertion in group CraL. Anatomically, intervertebral space in cranial lumbar or thoracic vertebral region is physiologically narrow than that in caudal lumbar region. The difficulty of catheterization may be enhanced when using midline approach in this region. The paramedian approach therefore was thought to be more feasible to be used for catheterization in group CraL.
Our results showed that although statistically not significant, the apparent difference in the time needed to identify the epidural space was caused by two dogs in group CraL in whom identification of the epidural space was time-consuming and took more than $2 \mathrm{~min}$, due to several redirections of the epidural needle. We thought it was attribute to the lack of obvious landmarks at cranial lumbar vertebral level, resulting in difficulty in determination of the predicted intervertebral space. However, since the total time from needle puncture to catheter placement and saline injection was comparable between two groups, indicating that less time was consumed of catheter insertion in group CraL. Our finding was also consistent with previous reports that epidural catheterization via the paramedian approach was faster, when it was compared with the midline approach [11]. One possibility was thought to be the steeper angle of entry of the paramedian epidural needle into the epidural space, facilitating catheter insertion $[2,11]$. In our study, we used paramedian approach to insert catheter in group CraL. The long axis of the needle was more parallel to the longitudinal axis of the spinal cord. In contrary, in group CauL, needle punctured according to the traditional method that needle penetrated into the skin and epidural space perpendicularly via the midline approach. Hence, it was speculated that less resistance might be encountered during introducing the catheter into the epidural space through the needle in group $\mathrm{CraL}$ than group CauL.

In the present study, regardless of epidural sites, catheter tip was located on the right side in more cases. We cannot find an exact reason to explain this result. Maybe it just occurred occasionally. In humans, it has already known that epidural catheters do not follow a straight and predictable course in the epidural during catheter insertion $[9,12]$. Presumably, any incidental factor during needle puncture and catheter insertion, or even the type of needle and catheter used may all affect the final location of catheter in the epidural space.

According to our evaluation criteria, except a mild subcutaneous bleeding occurred in 3 dogs receiving cranial lumbar epidural catheterization, we did not detect any macroscopic or microscopic injuries of selected spinal cord segments in both groups. In humans, it has known that multiple attempts are associated with complications such as trauma to neural structures $[1,5,14]$ and spinal haematoma [18]. Therefore it is reasonable to think that this subcutaneous bleeding was also caused by multiple puncture attempts, however, it seems inappropriate. In fact, in those 3 dogs, except one dog received the second epidural puncture, other two dogs was punctured only once. Redirecting needle subcutaneously, as well as multiple puncture attempts may both contribute to this mild trauma. Nevertheless under clinical circumstances, we do not consider mild subcutaneous bleeding would influence anesthesia and surgery considerably in patients with normal coagulation function.

Data of subjective evaluation showed the score of technical difficulty was slightly but statistically higher in group CraL than group CauL. As we mentioned above, we used 
standard experimental dog, in which the individual difference of body weight was minor. So it is unlikely that body weight affected the overall technical difficulty very much. Lack of obvious landmarks in cranial lumbar region might contribute to the higher subjective difficulty score in group CraL.

In conclusion, in the current study, we compared technique-related difficulty and safety of epidural catheterization between cranial and caudal lumbar segments. Timerelated results were comparable between two groups, and no obvious technique-related injuries of the spinal cord were found. Although the overall technical difficulty score was statistically higher in group CraL, we still considered that epidural catheterization in the cranial lumbar segments could be performed as feasibly and safely as that at the caudal lumbar vertebral segments in medium or large dogs in the clinical setting.

\section{REFERENCES}

1. Auroy, Y., Narchi, P. and Messiah, A. 1997. Serious complications related to regional anesthesia: results of a prospective survey in France. Anesthesiology 87: 479-486.

2. Blomberg, R. G., Jaanivald, A. and Walther, S. 1989. Advantages of the paramedian approach for lumbar epidural analgesia with catheter technique. A clinical comparison between midline and paramedian approaches. Anaesthesia. 44: 742-746.

3. Brodner, G., Van, Aken, H. and Gogarten, W. 2007. [Regional anesthesia for postoperative pain control]. Anasthesiol. Intensivmed. Notfallmed. Schmerzther. 42: 32-41 (in German with English abstract).

4. de Filho, G. R., Gomes, H. P. and da Fonseca, M. H. 2002. Predictors of successful neuraxial block: a prospective study. Eur. J. Anaesthesiol. 19: 447-451.

5. Horlocker, T. T., McGregor, D. G. and Matsushige, D. K. 1997. A retrospective review of 4767 consecutive spinal anesthetics: central nervous system complications. Perioperative Outcomes Group. Anesth. Analg. 84: 578-584.

6. Hotvedt, R., Platou, E. S. and Refsum, H. 1983. Electrophysiological effects of thoracic epidural analgesia in the dog heart in situ. Cardiovasc. Res. 17: 259-266.

7. Hotvedt, R., Platou, E. S. and Refsum, H. 1984. Effects of thoracic epidural analgesia on cardiovascular function and plasma concentration of free fatty acids and catecholamines in the dog. Acta Anaesthesiol. Scand. 28: 132-137.

8. Hotvedt, R., Refsum, H. and Platou, E. S. 1984. Cardiac electrophysiological and hemodynamic effects of betaadrenoceptor blockade and thoracic epidural analgesia in the dog. Anesth. Analg. 63: 817-824.

9. Hsin, S. T., Chang, F. C. and Tsou, M. Y. 2001. Inadvertent knotting of a thoracic epidural catheter. Acta Anaesthesiol. Scand. 45: 255-257.

10. Lee, C. J., Jeon, Y. and Lim, Y. J. 2007. The influence of neck flexion and extension on the distribution of contrast medium in the high thoracic epidural space. Anesth. Analg. 104: 15831586 .

11. Leeda, M., Stienstra, R. and Arbous, M. S. 2005. Lumbar epidural catheter insertion: the midline vs. the paramedian approach. Eur. J. Anaesthesiol. 22: 839-842.

12. Lim, Y. J., Bahk, J. H. and Ahn, W. S. 2002. Coiling of lumbar epidural catheters. Acta Anaesthesiol. Scand. 46: 603-606.

13. Lundberg, J., Biber, B. and Henriksson, B. A. 1991. Effects of thoracic epidural anesthesia and adrenoceptor blockade on the cardiovascular response to dopamine in the dog. Acta Anaesthesiol. Scand. 35: 359-365.

14. Puolakka, R., Haasio, J. and Pitkanen, M. T. 2000. Technical aspects and postoperative sequelae of spinal and epidural anesthesia: a prospective study of 3,230 orthopedic patients. Reg. Anesth. Pain Med. 25: 488-497.

15. Shores, A. 1993. Magnetic resonance imaging. Vet. Clin. North Am. Small Anim. Pract. 23: 437-459.

16. Skarda, R. T. and Tranquilli, W. J. 2007. Local and regional anesthetic and analgesic techniques: Dogs. pp. 561-593. In: LUMB \& JONES' Veterinary Anesthesia and Analgesia, 4th ed. (Tranquilli, W. J., Thurmon, J. C. and Grimm, K. A. eds.), Blackwell Publishing, Iowa.

17. Sprung, J., Bourke, D. L. and Grass, J. 1999. Predicting the difficult neuraxial block: a prospective study. Anesth. Analg. 89: 384-389.

18. Wulf, H. 1996. Epidural anaesthesia and spinal haematoma. Can. J. Anaesth. 43: 1260-1271. 